\title{
The Impact of Personal Interactions on the Experience of African-American Males on Multiracial Student Teams
}

\section{Ms. Kelly J Cross, Virginia Tech}

Ms. Cross earned her Bachelor's of Science in Chemical Engineering from Purdue University in 2007. She earned her Master's of Science in Materials Science and Engineering from the University of Cincinnati in 2011. Ms. Cross is currently completing her studies in the Engineering Education PhD program at Virginia Tech and involved with multiple educational research projects with faculty and graduate students. Her research interests include diversity and inclusion, teamwork and communication skills, assessment, and identity construction.

\section{Dr. Marie C Paretti, Virginia Tech}

Marie C. Paretti is an Associate Professor of Engineering Education at Virginia Tech, where she codirects the Virginia Tech Engineering Communications Center (VTECC). Her research focuses on communication in engineering design, interdisciplinary communication and collaboration, design education, and gender in engineering. She was awarded a CAREER grant from the National Science Foundation to study expert teaching in capstone design courses, and is co-PI on numerous NSF grants exploring communication, design, and identity in engineering. Drawing on theories of situated learning and identity development, her work includes studies on the teaching and learning of communication, effective teaching practices in design education, the effects of differing design pedagogies on retention and motivation, the dynamics of cross-disciplinary collaboration in both academic and industry design environments, and gender and identity in engineering. 


\title{
The Impact of Personal Interactions on the Experience of African-American Males on Multiracial Student Engineering Teams
}

\begin{abstract}
Team projects in undergraduate engineering programs are critical sites for professional skills development. Team projects allow students to try on professional roles as they interact with peers and faculty on projects designed to simulate engineering work. Also, engaging in engineering activities such as team projects can help students establish a sense of identity within their field, which in turn influences their persistence through college and into a career. Moreover, team projects intersect personal and professional identities and can be a source of conflict for students from populations that are traditionally underrepresented in engineering, such as women and ethnic minorities. While substantial research has identified challenges that women face on mixed-gender teams, few researchers have focused on the experiences of African-Americans on multiracial teams and this study aims to fill that gap.
\end{abstract}

This qualitative study employs a phenomenological approach, using a three-interview sequence with eight African-American male engineering students across academic levels (average age $=$ 19) as they worked on team projects at a large research-intensive, predominantly white institution (PWI). The project durations ranged from two weeks to two full semesters. The semistructured interviews 1) gathered background information about participants, 2) explored participant descriptions of the team functionality during the project, and 3) asked participants to reflect on the experience in the context of their engineering education and identities. Following the practices of phenomenology, all three interviews for each participant were grouped for analysis to holistically describe the "essence" of each participant's experience, which includes both textural (what) and structural (how) descriptions of the phenomenon.

Specifically, this paper highlights the impact of student personal interactions on the experience of African-American males on multiracial student teams in engineering. Based on the phenomenological analysis of a larger study, several units of meaning emerged across participants relative to their experience being an African-American male on a multiracial student team. However, this paper focuses exclusively on the emergent theme personal interactions. While most participants stated that their race did not impact their team experience, they did discuss feeling spotlighted in the larger community such as their department or university. The spotlighting included being singled out during a class lecture, being questioned for studying in the common area, getting "dirty looks" while walking through department buildings or on campus, and being stereotyped generally. As a result, students used multiple strategies to manage peer interactions they experienced during their multiracial student team. For example, the participants emphasized "getting to know" teammates outside of work. The implications of these emerging themes suggest that personal interactions directly influence how African-American males experience cross-race interaction on multiracial student teams. Interrogating the interactions African-American males experience within multiracial teams enhances our understanding of how they experience engineering and what peer interactions reduce spotlighting and disconnection. 


\section{Introduction}

Team projects in undergraduate engineering programs are critical sites for professional skills development. Designed to simulate engineering work, team projects allow students to try on professional roles as they interact with peers and faculty. Also, engaging in engineering activities such as a team project can help students establish a sense of identity within their field, which in turn influences their persistence through college and into a career. Moreover, team projects intersect personal and professional identities and can be a source of conflict for students from populations that are underrepresented in engineering, such as women and ethnic minorities. While substantial research has identified challenges that women face on mixed-gender teams, ${ }^{[1-}$

${ }^{4]}$ few researchers have focused on the experiences of African-Americans on multiracial teams. This study aims to fill that gap.

In previous studies, researchers have found that team projects in school provide students with the opportunity to interact in groups and practice professional teaming skills. As a result, the use of team projects to develop teaming skills has increased in engineering courses, and teamwork is now a central outcome required by accreditation agencies in the U.S. ${ }^{[5]}$. However, despite the emphasis of teamwork in undergraduate engineering education and the increasing prevalence of team projects, we have an underdeveloped understanding of team effectiveness and student team behaviors ${ }^{[6]}$.

We also know very little about multiracial student teams in engineering. However, research does show that cross-race interaction, which is a product of participating on a multiracial team, has an impact on student outcomes and perceptions. For example, the quantity of cross-race interactions has a statistically significant and positive correlation to multiple student outcomes ${ }^{[7]}$. Specifically, students who report less cross-race interaction also report lower gains in their knowledge of and ability to accept different races or cultures, general knowledge growth, critical thinking ability, and problem solving skill since entering college than their peers that reported higher levels of cross-race interaction ${ }^{[7]}$. As a result, observing the frequency of student crossrace interaction (voluntary and involuntary) can provide insight into interactions within multiracial student teams and students perceptions of their team experience.

Furthermore, while significant evidence suggests that increased cross-race interactions support a range of cognitive and social student outcomes, few studies have investigated the interaction quality or how interactions are experienced. For example, research on interracial relations indicates that Whites and ethnic minorities can have vastly different experiences while participating in the same cross-race interaction ${ }^{[8]}$. In other words, how African-American males perceive their experience on a multiracial student teams can be very different than their peers or White counterparts. Therefore, the guiding question for this analysis is as follows: How does personal interaction on a multiracial student team impact the experience of African-American male engineering students? 


\section{Methods}

To begin to bridge the gap in our understanding of the effects of race on students' team experiences, this study employed a phenomenological approach to understand the phenomenon of being an African-American male on a multiracial student team in engineering. This approach included a three-interview sequence with eight African-American male engineering students (across academic levels) as they worked on team projects at a large research-intensive, predominantly white institution (PWI).

\section{Participants}

The data reported in this paper is drawn from a larger study of interviews with 19 self-identified African-American engineering students across engineering disciplines. In this paper, we discuss the phenomenological analysis of interviews with the male participants $(n=8)$. The participants' departments included the following: Computer Science (CS), Mechanical Engineering (ME), Aerospace and Ocean Engineering (AOE), Biological System Engineering (BSE), Electrical Engineering (EE), Industrial and Systems Engineering (ISE), and undecided. The participants represent all undergraduate academic levels and represent a diverse pool of experience types and developmental stages (e.g., freshman design vs. senior design, assigned vs. self selected teammates, and mini two week vs. extended two semester projects).

\section{Data Collection and Analysis}

Data collection consisted of three individual interviews with each participant to understand his experience on a multiracial student team. Each three interview sequence was conducted over the course of a spring semester. In some cases, the team project was completed within this semester; but in other cases, the spring represented the continuation of a project begun in the fall (e.g., for a senior design class). The semi-structured interviews 1) gathered background information about participants, 2) explored participants' descriptions of the team functionality during the project, and 3) asked participants to reflect on the experience in the context of their engineering education and identities. Each interview was audio recorded and transcribed verbatim. Typically, the interviews lasted 45 minutes and each was conducted as least three weeks a part.

For each participant, data analysis followed the process outlined by Hycner ${ }^{[9]}$ and included the following general steps:

1. Bracket or block out personal views of the phenomenon of interest to minimize bias and reduce the data to individual significant statements that are relevant to the experience;

2. Group significant statements into larger "meaning units" or clusters;

3. Write textural description of "what" experienced;

4. Write structural description of "how" experienced;

5. Combine "what" and "how" into composite description of the experience or the phenomenological statement;

6. Perform a member check as validity technique where the themes, interpretations, and conclusions are tested with participants from whom the data were originally obtained. 
After completing the process for each participant, the clusters were compared across participants to identify common themes. A peer reviewer verified the results of the analysis after Step 2 and again after Step 3.

In this paper, we highlight the cluster of meaning, personal interactions that is directly related to student-student or peer interactions. Social science researchers define personal interactions as voluntary formal and informal interactions with team members ${ }^{[10]}$. The definition of personal interactions was operationalized in the current analysis as voluntary formal and informal interactions with team as a group, communication preferences that transpired during the team project, general perception of teammates (out-group), and personal characteristics that impact team dynamics.

\section{Results}

The male participants generally had a positive disposition toward teamwork and acknowledged how important teamwork is to engineering practice. At the same time, they described perceptions and experiences across their curricula that, consistent with the research noted earlier, inhibited their sense of belonging. Peer interactions, both within the team experience and as part of the larger culture, played a key role in either mitigating or reinforcing those barriers. Personal interaction was operationalized in the analysis as voluntary informal interactions with the team as a group that required self disclosure. Self-disclosure is the voluntary communication of intimate or personal information to another person ${ }^{[11,12]}$. Generally, personal interactions were cross-race interactions with the entire team or multiple members of the multiracial team of engineering students including statements about informal communication, judgments of how team members interacted with each other, and explanations of interpersonal behaviors that support team collaboration.

These personal interactions operated in two distinct ways: at the level of equality of ideas relative to the project itself (formal) and at the level of personal engagement outside the project work (informal). Below are a few representative statements that reflect this group of AfricanAmerican males. Note that the quotes are cited to be illustrative, not comprehensive.

Robert illustrated equal status within the project when he explained:

Robert: I guess we just like, didn't really have any problems while we were working, like we were all on the same kind of level, as far as the project went, so, it went pretty smoothly, is what I'm trying to say.

In this statement, Robert stated that his group had minimal conflict during the project and the personal interaction between him and the other teammates had gone smoothly. Also, his statement suggests the equal status of all members of his multiracial student team as they were "all on the same kind of level." Combined, these statements represent Robert's interpretation of his personal interaction with his overall team.

Jake expressed a similar sentiment when he described the context of his team's personal interaction and provided input to team decisions: 
Jake: This group is just a pretty straight forward, group project doesn't require too much of us, if we all work together, so let's just get it done and be on our way, so. Everybody, if anybody feels like this idea's not the best idea, I guess they will voice it and I, we all try to make sure that everybody's input is valued in the, like the final decisions.

According to Jake, the simplicity of the project didn't require complex relationships, communication or negotiation, but he did mention the way in which all opinions were respected. In other words, all opinions were recognized and appreciated within his multiracial student team. Later in the interview, Jake personalized his feelings when he emphasized the importance of being able to give his opinion or have a voice on his student team:

Jake: so, yeah I feel comfortable with my team members. I mean, everything, everybody's fairly cool. They ask me for inputs on like, what should we do, I mean, I'm kind of like make sure that everything is like the best it could be...

In these statements, Jake articulated the ease with which he communicated with teammates and felt free to engage. Furthermore, he went on to describe his role in verifying the work quality, which is an indication of his investment in the project and perspective of accountability. Combined these statements indicate Jake's comfort with self-disclosure to his team mates.

In addition to considering all members intellectual contributions, the participants described practicing effective personal interactions beyond the bounds of the project itself. Clay, for example, described such interaction as follows:

Clay: I think that it really helps, you to like get more comfortable around each other and get to know each other better. So, I think it is important for a team to be able to just step back and hang out without, without just doing work.

Interviewer: all right, OK, and so it kinda, does it kinda help you, to just to see them in different light, just to see them outside of work?

Clay: Definitely, cuz you know a lot of, when people are like working, they can act a lot differently, than if they are just sitting back comfortable. You really get to know a person a lot better like that.

In these statements, Clay emphasized learning about teammates in relaxed social setting can enhance the personal interaction within the multiracial student teams. "Getting to know" teammates better can provide context of teammate's perspective. According to Clay, some teammates may choose to communicate or behave differently in relaxed social settings than their normal professional interactions during team activities. In other words, a relaxed social setting can promote self-disclosure. Understanding the teammate as person is important to Clay as the "getting to know" is foundation for a closer relationship within a team. Clay emphasized close relationships with teammates when he stated:

Clay: I think trust is definitely a large, a very important, plays a very important role in teams. 
In this comment, Clay specifies trust between teammates as essential for a team process, but also supports his personal view of close relationships with members of his multiracial student team. The combination of Clay's quotes suggest that effective personal interactions on a multiracial student requires "getting to know" teammates beyond the work environment to reach some level of trust and potentially a close relationship. In other words, the informal communication and selfdisclosure either supported or was connected to the formal communication within the team.

\section{Discussion}

The results of the current study suggest personal interactions within a multiracial student team are significant to the experience of African-American males on multiracial student teams in engineering. The experiences of these participants highlighted the essential role of personal interaction among members of multiracial student team in engineering at both the project level and the social level. For example, Jake stressed his opinion being heard and valued in regards to team decisions. Also, Clay wanted to learn about teammates outside of work and specified trust as a key to any team project. Understanding personal interaction among multiracial student teams is particularly important in light of the significant potential for miscommunication based on nonverbal communication or subtle and unconscious bias that can lead to interracial distrust ${ }^{[13]}$. In other words, majority students may not recognize or acknowledge the cumulative effect subtle microaggressions (e.g., negative stereotypes of African-Americans in engineering) can have for African-American teammate, and these personal interactions and "getting to know" teammates can help mitigate those effects. The study results add credence to other recent studies that suggest familiarity as a feasible method to support students of color navigating academic culture in multiple ways ${ }^{[14]}$. For example, the personal interaction of establishing "trust" among team members led to more voluntary social interaction, which foster stronger peer-support groups ${ }^{\text {[15] }}$. Also, personal interactions are mediated by self disclosure. Self-disclosure is one mechanism to support the development of positive personal interactions among students on a multiracial student team and the development of cross-race friendships ${ }^{[16]}$. Finally, the result of this study supports other research that found that personal interactions, including engagement regulation, are a learned behavior ${ }^{[17]}$. In the current study, the participants learned to socialize with multiracial student team members outside of the project and these interactions definitely impacted their perceptions of their team experiences.

\section{Limitations}

As with all studies, there were limitations to results of the study. First, the study was conducted at a single site with a portion of the African-American male population. As a result, the results of the study are not meant to be generalized but understood within the context of the participant's perceptions of their experiences. A second limitation to the study is that only a single member of the multiracial student team was part of the study. Although personal interaction was the primary theme that emerged from the data, the perspectives of the other team members may have provided important context of the team experiences that the participants did not include. Finally, the data collection occurred over a single semester, which limited the time for social relationships to develop between. In other words, the change in relationships may not have been captured in the current study. Despite these limitations, the results of the analysis provide a 
deeper understanding of how engineering faculty can enhance the experience of AfricanAmerican males on multiracial student teams in engineering.

\section{Conclusion}

In conclusion, the multiracial student team experience was significant to the participants in terms of its impact on their academic experience. Specifically, personal interaction with teammates beyond the team activities supported this group of African-American males' positive perceptions of their multiracial student team experience in engineering. Importantly, the participants did not perceive their race as impacting their team experience but discussed being stereotyped in the larger community such as the department and university. Furthermore, the social norms of the larger community can reinforce privilege in engineering and decrease African-American student's sense of belonging. Additional themes that further characterize African-American males experiences on multiracial student teams including indifferent faculty interactions and the lack of friendship opportunities are discussed elsewhere. As a result, multiracial team projects should be monitored carefully to ensure positive experiences of all team members and to ascertain to what degree the team dynamics mimic the larger community or department culture within engineering.

\section{References}

1. Tonso, K.L., Teams that Work: Campus Culture, Engineer Identity, and Social Interactions. Journal of Engineering Education, 2006. 95(1): p. 25-37.

2. Okudan, G.1.E. and C.E. Mutluer. An Investigation on Information and Gender-Based Power in Product Design Decision-Making. in ASME 2007 International Design Engineering Technical Conferences and Computers and Information in Engineering Conference. 2007. American Society of Mechanical Engineers.

3. Tonso, K.L., On the outskirts of engineering : learning identity, gender, and power via engineering. 2007, Rotterdam: Sense Publishers.

4. Wolfe, J. and E. Powell, Biases in Interpersonal Communication: How Engineering Students Perceive Gender Typical Speech Acts in Teamwork. Journal of Engineering Education, 2009. 98(1): p. 5-16.

5. ABET, CRITERIA FOR ACCREDITING ENGINEERING PROGRAMS 2011-2012, A.A.B.f.E.a. Technology). Editor. 2010, ABET, Inc.: Baltimore, MD.

6. $\quad$ Borrego, M., et al., Team Effectiveness Theory from Industrial and Organizational Psychology Applied to Engineering Student Project Teams: A Research Review. Journal of Engineering Education, 2013. 102(4): p. $472-512$.

7. Chang, M.J., et al., The Educational Benefits of Sustaining Cross-Racial Interaction among Undergraduates. The Journal of Higher Education, 2006. 77(3): p. 430-455.

8. Shelton, J.N., J.A. Richeson, and J. Salvatore, Expecting To Be the Target of Prejudice: Implications for Interethnic Interactions. Personality and Social Psychology Bulletin, 2005. 31(9): p. 1189-1202.

9. Hycner, R., Some guidelines for the phenomenological analysis of interview data. Human Studies, 1985. 8(3): p. 279-303.

10. Dovidio, J.F., S.L. Gaertner, and K. Kawakami, Intergroup Contact: The Past, Present, and the Future. Group Processes \& Intergroup Relations, 2003. 6(1): p. 5-21.

11. Turner, R.N., M. Hewstone, and A. Voci, Reducing explicit and implicit outgroup prejudice via direct and extended contact: The mediating role of self-disclosure and intergroup anxiety. Journal of Personality and Social Psychology, 2007. 93(3): p. 369-388.

12. Miller, N., Personalization and the promise of contact theory. Journal of Social Issues, 2002. 58(2): p. 387410. 
13. Dovidio, J.F., et al., Why can't we just get along? Interpersonal biases and interracial distrust. Cultural Diversity and Ethnic Minority Psychology, 2002. 8(2): p. 88-102.

14. Harper, S.R., Am I my brother's teacher? Black undergraduates, racial socialization, and peer pedagogies in predominantly white postsecondary contexts. Review of Research in Education, 2013. 37(1): p. 183-211.

15. Hrabowski, F.A. and W. Pearson Jr, Recruiting and Retaining Talented African-American Males in College Science and Engineering. Journal of College Science Teaching, 1993. 22(4): p. 234-38.

16. Davies, K., S.C. Wright, and A. Aron, Cross-group friendships: How interpersonal connections encourage positive intergroup attitudes, in Moving beyond prejudice reduction: Pathways to positive intergroup relations, L.R.T.R.K. Mallett, Editor. 2011, American Psychological Association: Washington, DC, US. p. 119-138.

17. Hall, W.D., A.F. Cabrera, and J.F. Milem, A tale of two groups: Differences between minority students and non-minority students in their predispositions to and engagement with diverse peers at a predominantly White institution. Research in Higher Education, 2011. 52(4): p. 420-439. 